\begin{tabular}{|c|c|}
\hline & $\begin{array}{l}\text { International Journal of Trend in Scientific } \\
\text { Research and Development (IJTSRD) }\end{array}$ \\
\hline 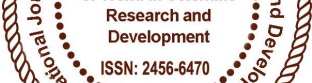 & International Open Access Journal \\
\hline 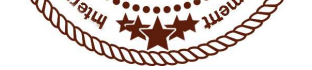 & ISSN No: 2456 - 6470 | www.ijtsrd.com | Volume - 2 | Issue - 2 \\
\hline
\end{tabular}

\title{
A Case Study on Elastic Oscillationsand its Applications in Bungee Jumping by Differential Equations
}

Gowri. P

Asst. Prof, Department of Mathematics, Sri Krishna Arts and Science College, Coimbatore, Tamil Nadu, India
Glory Bebina. E

III Bsc Maths, Sri Krishna Arts and Science College, Coimbatore, Tamil Nadu, India

\section{ABSTRACT}

The aim of this project is to develop an understanding of the mathematics and physics involved in a bungee jump, in order to gain a better understanding of the concepts involved. We will use Newton's law and utilize techniques needed to solve second order differential equations. For simplicity; we will regard the jumper as a point mass in one dimension and we will make the assumption that wind resistance has a linear relationship to speed. We will also assume that the bungee cord obeys Hooke's laws and doesn't break.

Keywords: Newton's second law; Hooke's law; Young's modulus; Wind resistance; Driving force; Terminal velocity.

\section{Modeling the Motion}

With a bungee jump, the model of the motion in one dimension with respect to time can be broken into two different sections: the free fall from the ledge and the time when the bungee cord is pulling on the jumper. To model both sections we need Newton's second law of motion. It says that the total of all the forces acting on an object is equal the mass of the object multiplied by its acceleration.

$\Sigma \mathrm{F}=\mathrm{ma}$

To describe how the bungee cord will pull on a bungee jumper we will use Hooke's law.Hooke's law says that the force exerted by a spring is proportional to the distance that it has been stretched from its equilibrium point. The relationship is said to be $\mathrm{F}=-$ $\mathrm{kx}$, where $\mathrm{k}$ is a constant that is specific to the spring. The negative is in the equation so that the force is in opposite direction of the stretch (pushing/pulling toward the resting point of the spring). For our model, we assume that a bungee cord will behave like aspring described by Hooke's law. The spring constant $\mathrm{k}$ can be found experimentally by hanging a known weight on a spring and measuring how far it stretches. also, $\mathrm{k}$ can be found by rearranging the Young's modulus formula. The Young's modulus formula applies to objects that have a constant cross-sectional area (same thickness throughout) and objects that have the same material all throughout, the formula also only applies for forces that compress or stretch the object. The formula says that any material has constant that relates the stress on an object to the strain on the object. The stress is said to be the force applied on the object divided by the cross-sectional area of the object, F/A. The strain is said to be the change in length divided by the initial length, $\mathrm{dL} / \mathrm{L}$. The Young's modulus formula is:

$$
\mathrm{Y}=\frac{F / A}{d L / L}
$$

where $\mathrm{Y}$ is the Young's modulus, a constant for a given material. $\mathrm{F}$ is the force being applied, $\mathrm{A}$ is the cross-sectional area. L is the length of unstretched cord, and dL is the change in the length of the cord once it has been stretched. This can be rearranged to look like Hooke's law, if we remember that $\mathrm{dL}$ and the $\mathrm{x}$ from hook's law have the same meaning. 


$$
\mathrm{F}=\frac{A Y}{L} x
$$

The Young's modulus constant, the cross-sectional area, and unstretched cord length will not change for any given cord, so we can say that the spring constant in Hooke's law is:

$$
\mathrm{k}=\frac{A Y}{L}(1)
$$

In order to keep the math doable, we will also stick with a wind resistance that is linearly proportional to the velocity. There are more accurate and complicated formulas to account for wind resistance, but for this paper we will be using:

$$
F_{\text {wind }}=-\beta v
$$

In this paper, we will define the positive $\mathrm{x}$ direction to be going down.

\section{Free fall}

When the bungee jumper first jumps off of the bridge, they will have the forces of gravity and wind resistance acting on them. The force of gravity is positive because we've defined the positive $\mathrm{x}$ direction to be going down. The wind resistance is $-\beta v$ because the force of wind resistance is always in the opposite direction of the velocity and because we decided that making the force of wind resistance proportional to velocity was appropriate for this paper. Using Newton's second law:

$\mathrm{mg}-\beta v=\mathrm{ma}$

using the fact that $\mathrm{a}=\mathrm{dv} / \mathrm{dt} ; \mathrm{v}=\mathrm{dx} / \mathrm{dt}$ and a little algebra, we can put this into the form of an inhomogeneous equation.

$$
\frac{d^{2} x}{d t^{2}}+\frac{\beta}{m} \frac{d x}{d t}=g
$$

\section{Solving the homogeneous equation}

To find the general solution of the inhomogeneous equation, we must first find the solutions to the equivalent homogeneous equation. In other words, we will set the driving force $g$ to zero. (Note: there are other easier ways, but we will solve our inhomogeneous equation this way so that the process is the same as the process needed in the next section.)

$$
\frac{d^{2} x}{d t^{2}}+\frac{\beta}{m} \frac{d x}{d t}=0
$$

The characteristic equation for the homogeneous equation is:

$$
\mathrm{p}(\lambda)=\lambda^{2}+\frac{\beta}{m} \lambda
$$

We set $p(\lambda)$ to 0 to find that the roots of the equation are:

$$
\lambda_{1}=0, \lambda_{2}=-\frac{\beta}{m}
$$

The solution to homogeneous equation is:

$$
x_{h}=C_{1} e^{\lambda_{1} t}+C_{2} e^{\lambda_{2} t}
$$

Substituting in our values for

$$
x_{h}=C_{1}+C_{2} e^{-\frac{\beta t}{m}}
$$

\section{Solving for the particular solution}

Now that the homogeneous equation has been solved, the only thing left to find is the particular solution. If we look at equation (2) and we look at the driving force that is on the right-hand side, then we see that the driving force is a polynomial of zero'thdegree (a constant). The particular solution to the equation should be a polynomial. If we say a particular solution is:

$$
x_{p}=\mathrm{at}+\mathrm{b}, \frac{d}{d t} x_{p}=a \text { and } \frac{d^{2} x}{d t^{2}} x_{p}=0
$$

Then we can plug this into (2) and solve for a

$$
\begin{aligned}
(0)+\frac{\beta}{m}(a) & =g \\
\frac{\beta}{m}(a) & =g
\end{aligned}
$$

$$
a=\frac{m g}{\beta}
$$

Our general solution is:

$x=C_{1}+C_{2} e^{-\frac{\beta t}{m}}+\frac{m g}{\beta} t+\mathrm{b}$ 
$C_{1}$ andb are both constants. because $C_{1}$ is an arbitrary constant, we say that it

accounts for $b$.

$x=C_{1}+C_{2} e^{-\frac{\beta t}{m}}+\frac{m g}{\beta} t$

\section{Below the equilibrium point for the cord}

So far, an equation for the motion of the bungee jumper has been found. Now, we need to find an equation of motion for when the bungee jumper is below the equilibrium point. To do this we look back to Newton's second law:

$\Sigma \mathrm{F}=\mathrm{ma}$

When the jumper is below the equilibrium point, they still have gravity and wind resistance acting on them. But, now they also have the force of the cord pulling on them. We will say that the force applied by the bungee cordis-kx (from Hooke's law). Using this with Newton's second law:

$$
\mathrm{mg}-\beta \frac{d x}{d t}-k x=m \frac{d^{2} x}{d t^{2}}
$$

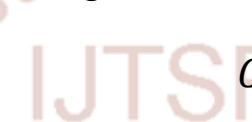

\section{Case 2}

Once again, this need to be rearranged algebraically to look like an inhomogeneous equation

$$
\frac{d^{2} x}{d t^{2}}+\frac{\beta}{m} \frac{d x}{d t}+\frac{k}{m} x=g
$$

\section{Solving the homogeneous equation}

The first step to solving the inhomogeneous equation is to solve the equivalent homogeneous equation.

$$
\frac{d^{2} x}{d t^{2}}+\frac{\beta}{m} \frac{d x}{d t}+\frac{k}{m} x=0
$$

The characteristic equation is

$$
\mathrm{P}(\lambda)=\lambda^{2}+\frac{\beta}{m} \lambda+\frac{k}{m}
$$

We set $\mathrm{P}(\lambda)$ to 0 and use the quadratic to find that the roots of the characteristic equation are:

$$
\lambda=\frac{-\beta / m \pm \sqrt{(\beta / m)^{2}-4(k / m)}}{2}
$$

At this point, the solution to the homogeneous equation can go in three directions, depending on the discriminant. There are three cases, when $(\beta / m)^{2}-4(k / m)$ is greater than zero, equal to zero, or less than zero.

\section{Case 1}

$$
(\beta / m)^{2}-4\left(\frac{k}{m}\right)>0
$$

This is said to be over damped, and the solution will not oscillate. If bungee cord

and jumper system fall into this category then there is a problem, because the bungee jumper will likely experience too much acceleration. In this case, the characteristic equation has two distinct real roots. The solution will take the form,

$$
x_{h}=C_{1} e^{\lambda_{1} t}+C_{2} e^{\lambda_{2} t}
$$

We can plug in our expression for $\lambda$ to get,

$x_{h}=C_{1} e^{\left(-\beta / m \pm \sqrt{\left.(\beta / m)^{2}-4(k / m)\right)} t / 2\right.}+$

$C_{2} e^{\left(-\beta / m \pm \sqrt{\left.(\beta / m)^{2}-4(k / m)\right)} t / 2\right.}$

This case is said to be critically damped, and its solution appears very similar to the over damped case. The characteristic equation has one real root, and the solution will have the form.

Or

$$
(\beta / m)^{2}-4(k / m)=0
$$

30 $6470 x_{h}=C_{1} e^{\lambda t}+C_{2} t e^{\lambda t}$

$$
x_{h}=\left(C_{1}+C_{2} t\right) e^{\lambda t}
$$

Remembering that in this case

$(\beta / m)^{2}-4(k / m)=0$, we say that $\lambda=-\beta / 2 m$ and thatour solution is,

\section{Case 3}

$$
(\beta / m)^{2}-4\left(\frac{k}{m}\right)<0
$$

This case is said to be under damped, and it's solution oscillates. The solution in this case will be a combination of the real and imaginary parts of $e^{\lambda t}$. To find this, we need to know what the real and imaginary parts of $e^{(a+i b) t}$ are. To do this we first split up the exponent. 
International Journal of Trend in Scientific Research and Development (IJTSRD) ISSN: 2456-6470

$$
e^{(a+i) t}=e^{a t} e^{(b i) t}
$$

$e^{b i t}$ splits up into $\cos b t+i \sin b t$, so we get:

$$
\begin{aligned}
& e^{a t} e^{(b i) t}=e^{a t}(\cos b t+i \sin b t) \\
& \left.=e^{a t} \cos b t+i e^{a t} \sin b t\right)
\end{aligned}
$$

The real part of the equation above is $e^{a t} \operatorname{cosbt}$ while the imaginary part is $e^{a t} \sin$ bt. If plug in the $\lambda$ value that we got from equation (4), and we use the same process to separate out the real and imaginary part, then we get that the real part of $e^{\lambda} \mathrm{t}$ is:

$$
e^{-\beta t / 2 m} \cos t \frac{\sqrt{\left|(\beta / m)^{2}-4(k / m)\right|}}{2}
$$

While the imaginary part is:

$$
e^{-\beta t / 2 m} \sin t \frac{\sqrt{\left|(\beta / m)^{2}-4(k / m)\right|}}{2}
$$

The homogeneous solution will be a combination of the real and imaginary parts of $e^{\lambda t}$

$$
\begin{aligned}
& x_{h} \\
& =C_{1} e^{-\frac{\beta t}{2 m}} \cos t \frac{\sqrt{\left|(\beta / m)^{2}-4(k / m)\right|}}{2} \\
& +C_{2} e^{-\frac{\beta t}{2 m}} \sin t \frac{\sqrt{\left|(\beta / m)^{2}-4(k / m)\right|}}{2}
\end{aligned}
$$

Because there is no $t$ term the coefficient a must be zero and as a result:

$$
\frac{k}{m} b=g
$$

$\mathrm{b}=\frac{g m}{k}$

So the particular solution is:

$$
x_{p}=\frac{g m}{k}
$$

\section{PROBLEMS}

\section{Solving without a numerical solverSetting variables}

Before we can take the last steps to solving for the position as a function of time, we need some initial conditions and parameters. We are going to model an $80 \mathrm{~kg}$ man jumping of a bridge with a height of $100 \mathrm{~m}$ with a 30 meter long bungee cord with adiameter of .00635 meters (quarter inch).

We measured a bungee cord that had the same thickness and a length of $1.31 \mathrm{~m}$ and found that when a force of $10 \mathrm{~N}$ was applied the cord stretched $.16 \mathrm{~m}$. The bungee cordcould stretch to a maximum of 220

\section{SOLUTION}

\section{Solving for the particular solution}

The next step is to get the particular solution of the inhomogeneous equation.

$$
\frac{d^{2} x}{d t^{2}}+\frac{\beta}{m} \frac{d x}{d t}+\frac{k}{m} x=g
$$

Fortunately, the particular solution does not have multiple cases to consider. In addition, the driving force is a polynomial of zero'th degree just like it was for the free fall equation. We can assume that the solution is some polynomial with unknown coefficients and then plug the solution into the equation above to find those coefficients.

$$
x_{p}=a x+b, \quad \frac{d}{d t} x_{p}=a, \quad \frac{d^{2}}{d t^{2}} x_{p}=0
$$

plugging these into the inhomogeneous equation we get,

(0) $+\frac{\beta}{m} a+\frac{k}{m}(a x+b)=g$

$$
\mathrm{Y}=\frac{\mathrm{F} / \mathrm{A}}{\mathrm{dL} / \mathrm{L}}
$$

$$
\mathrm{Y}=\frac{10\left(\pi(0.00635 / 2)^{2}\right.}{0.16 / 1.31}
$$$$
=2.59 \times 10^{6}
$$

We will assume that the cord that we measured is suitable for bungee jumping. We will also assume that they could will stretch linearly with the force applied so that we can use the Young's modulus as well as Hooke's law. With these assumptions the $\mathrm{k}$ value that is used in Hooke's law will now be determined.

Pulling from equation (1):

$\mathrm{K}=\frac{A Y}{L}$

$$
\mathrm{K}=\frac{\left(\pi(0.00635)^{2}\right) 2.59 \times 10^{6}}{30}
$$

$\mathrm{K}=2.73$ 
Now we have all but one variable, $\beta$. To get this variable we can solve for based on accepted values for the terminal velocity of a person. To do this, we go back to

$\sum F=$ ma and look at free fall.

$\mathrm{mg}-\beta \mathrm{v}=\mathrm{m} \frac{d v}{d t}$

Because we looking at when the person has reached terminal velocity (no change invelocity), we can set $\mathrm{dv}=\mathrm{dt}$ to zero and solve for $\beta$.

$\mathrm{mg}-\beta \mathrm{v}=0$

$\beta=\frac{m g}{v}$

In solving for $\beta$ we had already assumed that the person was at terminal velocity, so we can plug in the value that we looked up for v. According to hypertextbook.com, a good approximate of the terminal velocity of a person is $60 \mathrm{~m} / \mathrm{s}$. with this we get:

$\beta=\frac{(80)(9.81)}{60}$

$\beta=13.08$

\section{RESULT}

Let's replace $-\beta / \mathrm{m}$ with $A$ to make the next few steps easier

$\mathrm{x}(\mathrm{t})=C_{1}+C_{2} e^{-A}+\frac{m g}{\beta} t$

Differentiating we get,

$$
\frac{d}{d t} x(t)=v(t)=-C_{2} A e^{-A}+\frac{m g}{\beta}
$$

If we use the initial conditions

$\mathrm{x}(0)=C_{1}+C_{2} e^{-A(0)}+\frac{m g}{\beta}(0)$

$\mathrm{v}(0)=-C_{2} A e^{-A(0)}+\frac{m g}{\beta}$

This simplifies to,

$\mathrm{x}(0)=C_{1}+C_{2}$

$\mathrm{v}(0)=-A C_{2}+\frac{m g}{\beta}$

Solving for $C_{1}$ and $C_{2}$

$$
\begin{aligned}
& \text { Chand } \quad C_{1}=x(0)-C_{2} \\
& C_{2}=-\frac{v(0)-(m g) /(\beta))}{A}
\end{aligned}
$$

YOUNG'S MODULUS $(\mathrm{Y})=2.59 \times 10^{6}$

HOOK'S CONSTANT $(\mathrm{K})=2.73$

$\operatorname{VARIABLE}(\beta)=13.08$

\section{At the start of the jump}

Now that we have all of the variables, we can use the use the general solutions that we found earlier. First we will use the initial conditions of $x(0)=-30(30 \mathrm{~m}$ above the equilibrium point of the unstretched cord) and $\mathrm{v}(0)=0$. Since we are using $\mathrm{x}(0)$ and $\mathrm{y}(0)$, the initial $t$ value has to be zero. We have the equation (3) with the unknowns $C_{1}$ and $C_{2}$. To solve for both unknowns we will have to use equation (3) and its derivative.

\section{SOLUTION}

$\mathrm{x}(\mathrm{t})=C_{1}+C_{2} e^{-\frac{\beta t}{m}}+\frac{m g}{\beta} t$

$$
C_{1}=x(0)+\frac{m(v(0)-(m g) /(\beta))}{\beta}
$$

$$
C_{2}=-\frac{m(v(0)-(m g) /(\beta))}{\beta}
$$

If we plug in the known values for our parameters and conditions

$$
C_{1}=-30+\frac{80(0-(80)(9.81) /(13.08))}{13.08}
$$

$C_{2}=-\frac{80(0-(80)(9.81) /(13.08))}{13.08}$

From this we get that $C_{1}=-396: 97$ and that $C_{2}=$ 366:97. So, the equation for free fall becomes

$\mathrm{x}(\mathrm{t})=366.97 e^{-0.1635 t}+60 t-396.97$ 
From this position function we need two things. First, we need to find when $\mathrm{x}$ is greater than zero because this is when we need a new equation to represent the bungee jumper. Second, we need to find the acceleration as a function of time. From the graph of the position function it seems that $\mathrm{x}(\mathrm{t})=0$ when $\mathrm{t}=$ 2.67. If we plug this and all of our knowns into the velocity function:

$\mathrm{v}(\mathrm{t})=-C_{2} A e^{-A t}+\frac{m g}{\beta}$

Then we get that $\mathrm{v}(2.67)=21.22$. We will need this velocity as well as $t=2.67$ for the initial conditions of the next equation.

\section{RESULT}

$\operatorname{TIME}(\mathrm{t})=2.67$

VELOCITY $\mathrm{v}(2.67)=21.22$

\section{After the cord starts to pull}

To solve this part of the situation we still use the same parameters, but different initial conditions and equations. To make the solution easier to find we will say that the time $2.67 \mathrm{~s}$ for the previous equation is at $0 \mathrm{~s}$ for the new equation. The position and velocity stay unchanged, however. For this part of problem, we need to decide which case to use. If we plug known values into $(\beta / m)^{2}-4(\mathrm{k} / \mathrm{m})$ then we get 0.1098 which means that we must use case 3 . Our solution for $\mathrm{x}$ is a combination of both the solution in case three and the particular solution.

\section{SOLUTION}

$$
\begin{aligned}
& \mathrm{x}(\mathrm{t})= \\
& \begin{array}{l}
C_{1} e^{-\frac{\beta t}{2 m}} \cos t \frac{\sqrt{\left|(\beta / m)^{2}-4(k / m)\right|}}{2}+ \\
\quad C_{2} e^{-\frac{\beta t}{2 m}} \sin t \frac{\sqrt{\left|(\beta / m)^{2}-4(k / m)\right|}}{2}+\frac{g m}{k}
\end{array}
\end{aligned}
$$

This is a lot easier to deal with if we let

$\mathrm{A}=\beta / 2 \mathrm{~m}$ and

$\mathrm{B}=\left(\operatorname{sqrt}\left|(\beta / m)^{2}-4(\mathrm{k} / \mathrm{m})\right|\right) / 2$

$$
\mathrm{x}(\mathrm{t})=C_{1} e^{-A t} \cos (B t)+C_{2} e^{-A t} \sin (B t)+\frac{g m}{k}
$$

We differentiate this and get that:

$$
\mathrm{v}(\mathrm{t})=C_{1}\left(-A e^{-A t} \cos (B t)-\right.
$$

$B e-A t \sin B t)+C 2(-A e-A t \sin B t+B e-A t \cos B t)$

If we put $\mathrm{x}$ and $\mathrm{v}$ in terms of an initial condition then we get

$\mathrm{x}\left(t_{i}\right)=C_{1} e^{-A t_{i}} \cos \left(B t_{i}\right)+C_{2} e^{-A t_{i}} \sin \left(B t_{i}\right)$

$\mathrm{v}\left(t_{i}\right)=C_{1}\left(-A e^{-A t_{i}} \cos \left(B t_{i}\right)-\right.$

$B e-A t i \sin B t i)+C 2(-A e-A t i \sin B t i+$

$B e-A t i \cos B t i)$

Now we can put this into a matrix and row reduce

$$
\begin{array}{ccc}
e^{-A t_{i}} \cos \left(B t_{i}\right) & e^{-A t_{i}} \sin \left(B t_{i}\right) & \mathrm{x}\left(t_{i}\right) \\
\left(-A e^{-A t_{i}} \cos \left(B t_{i}\right)-B e^{-A t_{i}} \sin \left(B t_{i} e^{-A i t}\right)\right) & \left(-A e^{-A t_{i}} \sin \left(B t_{i}\right)+B e^{-A t_{i}} \cos \left(B t_{i}\right)\right) & \mathrm{v}\left(t_{i}\right)
\end{array}
$$

From this we get that $C_{1}=-315: 94$ and that $C_{2}=-$ 168.61. The position function after the bungee jumper has gone past the equilibrium point of the cord is $\mathrm{x}(\mathrm{t})$ $315.94 e^{-0.16} \cos (0.1636 t)-$
$168.61 e^{-0.1635} \sin (0.1636 t)+287.5603$

Alternatively, we could have made solving for the initial conditions on the second equation easier by saying it started at time zero. The constants $C_{1}$ and $C_{2}$ would turn out different, and the $t$ would have to be replaced with $\mathrm{t}-2.67$. The equation would still have the same time range. This turns out to be easier because all of exponential terms go to 1, all of the sine terms go to zero, and all of the cosine terms go to 1 .

\section{RESULT}

$C_{1}=-315.94$

$C_{2}=-168.61$

$\mathrm{x}(\mathrm{t})=$

$315.94 e^{-0.1635 t} \cos (0.1636 t)-$

$168.61 e^{-0.16} \sin (0.1636 t)+287.5603$ 
4. A cord s $20 \mathrm{~m}$ long un-stretched if its elasticity is $50 \%$ and the spring constant is $20 \mathrm{~N} / \mathrm{m}$, what is the maximum force that can he held?

\section{SOLUTION}

Use the formula $\mathrm{F}=\mathrm{kx}$

$\mathrm{X}$ is calculated by taking $50 \%$ of theun-stretched cord. Therefore $\mathrm{x}=10 \mathrm{~m}$. simply plug the values into the formula.

$\mathrm{F}=(20 \mathrm{~N} / \mathrm{m})(10 \mathrm{~m})$

$\mathrm{F}=200 \mathrm{~N}$

\section{RESULT}

This is the maximum force that the cord can hold is $200 \mathrm{~N}$.

\section{Using a Numerical Solver to find the Motion of the Jumper}

In order to get the correct motion of the jumper, we need to find a way to model the position as they move up and down due to the bungee cord after they leap. Using the origin as the equilibrium point of the weighted bungee, when $x>0$ the motion of the jumper can be modeled as the sum of the forces equals the mass multiplied by the acceleration. To model the oscillation, we will treat the bungee cord as a spring, and employ Hooke's Law to model the force of the bungee on the jumper. Hooke's Law is used for calculating the force imparted by a spring on an attached object and is defined as $\mathrm{F}=\mathrm{kx}$. where $\mathrm{x}$ is the displacement from the force. The spring constant $\mathrm{k}$ is given by dividing the force in Newtons by the displacement caused by that amount of force.

There are three forces acting on the jumper when they are under the influence of the bungee. First, is the force of gravity, which is simply $\mathrm{mg}$. Second, there is the force due to the bungee, which is modeled after a spring with a force $\mathrm{F}=-\mathrm{kx}$ with $\mathrm{k}$ being the spring constant of the bungee cord. Third, the force of wind resistance, with a wind resistance constant $\beta$, whose value is determined above in equation (??), which has a linear relationship with the velocity of the jumper. The calculations involved in finding the true value for $\beta$ are rather complicated and beyond the scope of our understanding, so we will use the calculated constant. Adding up the forces, we get
Where - $\mathrm{kx}$ is the force of the bungee on the jumper, and $\beta \mathrm{v}$ is the force of the wind resistance. It makes sense that as the magnitudes of $\mathrm{kx}$ and $\beta \mathrm{v}$ increase, they will eventually overcome the force of gravity. When this happens the jumper will be at the end of the downward motion of the oscillation, and will be shot back up from the force of the bungee.

\section{Finding the Equation of Motion}

In order to find an equation of the motion of the jumper, the equation above must be broken down to components that make up each force.

$\mathrm{mg}-\mathrm{kx}-\beta \mathrm{v}=\mathrm{ma}$

Moving the terms around, we get the expression

$$
x^{\prime \prime}+\frac{\beta}{m} x^{\prime}+\frac{k}{m} x=g
$$

This gives a differential equation with a forcing term g, which can be solved with

relative ease. In order to utilize MATLAB's pplane8

utility, we must have two first order differential equations to relate to one another. These equations were found using the following steps:

substituting $\mathrm{a}=\beta / \mathrm{m}$ and $\mathrm{b}=\mathrm{k} / \mathrm{m}$, and isolating the $x^{\prime \prime}$ we get

$$
x^{\prime \prime}=g-a x^{\prime}-b x
$$

Now, the next step is to create two first order equations.

$$
\begin{gathered}
y_{1}=x \\
y_{2}=x^{\prime}
\end{gathered}
$$

Now we plug $y_{1}$ and $y_{2}$ into our equation, with the addition of $y_{1}^{\prime}=y_{2}$

$$
\begin{gathered}
y_{1}^{\prime}=y_{2} \\
y_{2}^{\prime}=g-a y_{2}-b y_{1}
\end{gathered}
$$

When $\mathrm{a}$ and $\mathrm{b}$ are substituted back into the equation we get,

$$
\begin{gathered}
y_{1}^{\prime}=y_{2} \\
y_{2}^{\prime}=g-\frac{\beta}{m} y_{2}-\frac{k}{m} y_{1}
\end{gathered}
$$

$$
F_{\text {gravity }}-k x-\beta v=F_{\text {net }}
$$


International Journal of Trend in Scientific Research and Development (IJTSRD) ISSN: 2456-6470

RESULT:

$$
\begin{gathered}
y_{1}^{\prime}=y_{2} \\
y_{2}^{\prime}=g-\frac{\beta}{m} y_{2}-\frac{k}{m} y_{1}
\end{gathered}
$$

\section{REFERENCES}

1. Gowri.P, Abhinandana.R, MadhuGokul.B, Saranya.B, Mrithula.R" A Case Study on path ofProjectile Motion and its Application", International journal of Engineering Science Invention research and Development, vol-4, Issue4, Dec-2017.

2. Gowri.P, Deepika.D and Krithika.S, "A Case study on Simple Harmonic Motion and its Applications", International Journal of Latest Engineering and $\quad$ Management Research(IJLEMR).

3. Adkins, Samantha, Katy Arthur, Je_ Bene_eld, and Brian Boling. "A MathematicalRepresentation of the Motion of a Bungee Jumper." Okstate.edu. Oklahoma State University, 14 Oct. 2002. Web. 20 Feb. 2014.

4. Polking, John, Albert Boggess, and David Arnold. Di erential Equations. 2nd ed. Upper Saddle River: Pearson Education, 2005. Print.
5. Kelly, Nicole. "Modeling a Bungee Jump." Fvcc.edu. Flathead Valley Community College, 17 May 2013. Web. 21 Feb. 2014.

6. Serway, Raymond A, John W. Jewett. Physics for Scientists and Engineers.9thed. Cengage Learning, 2014. Print.

7. Priyadharsini.S, Gowri,P, Aparna.T,'Solution of Fuzzy Integrodifferential Equation by Fuzzy Laplace Transform Method", International Journal of Advanced Mathematics V.I, N.1, 2017, pp.

8. Differential equations with Boundary Value Problems polking, bogges, Arnold

9. Pendulum Shifts to Active ETFs www.etftrends.com

10. Mathematical swingers: The Simple Pendulum as a Log Application www.http://my execp.com 